\title{
Complete Solution for Quasicrystals
}

\author{
Antony J. Bourdillon \\ UHRL, San Jose, CA, USA \\ Email: bourdillona@sbcglobal.net
}

How to cite this paper: Bourdillon, A.J. (2020) Complete Solution for Quasicrystals. Journal of Modern Physics, 11, 581-592. https://doi.org/10.4236/jmp.2020.114038

Received: March 25, 2020

Accepted: April 21, 2020

Published: April 24, 2020

Copyright $\odot 2020$ by author(s) and Scientific Research Publishing Inc. This work is licensed under the Creative Commons Attribution International License (CC BY 4.0).

http://creativecommons.org/licenses/by/4.0/

\begin{abstract}
A set of discoveries are described that complete the structural model and diffraction theory for quasicrystals. The irrational diffraction indices critically oppose Bragg diffraction. We analyze them as partly rational; while the irrational part determines the metric that is necessary for measurement. The measurement is verified by consistency with the measured lattice parameter, now corrected with the metric and index. There is translational symmetry and it is hierarchic, as is demonstrated by phase-contrast, optimum-defocus imaging. In Bragg's law, orders are integral, periodic and harmonic; we demonstrate harmonic quasi-Bloch waves despite the diffraction in irrational, geometric series. The harmonicity is both local and long range. A breakthrough in understanding came from a modified structure factor that features independence from scattering angle. Diffraction is found to occur at a given "quasi-Bragg condition" that depends on the special metric. This is now analyzed and measured and verified: the metric function is derived from the irrational part of the index in three dimensions. The inverse of the function is exactly equal to the metric that was first discovered independently by means of "quasi-structure factors". These are consistent with all structural measurements, including diffraction by the quasicrystal, and with the measured lattice parameter.
\end{abstract}

\section{Keywords}

Quasicrystal, Icosahedra, Hierarchic, Integral, Periodic, Harmonic, Irrational, Geometric Series, Metric

\section{Introducing Harmonics in Hierarchy}

"A metallic phase with long range order but with no translational symmetry" [1], how come? The greater the prize, the worse the gaff: the translational symmetry is strictly hierarchic [2]-[15]. How, in particular, can the diffraction represent long range order when it occurs as irrational numbers, aperiodically ordered, and in geometric series? Since Bohr's atom, all of modern wave physics 
have required harmonic solutions; how does that strange series harmonize? This study shows precisely how the exceptional harmony occurs.

The original solid-state phase was discovered in 1982 and published in 1984 [1], shortly before my hierarchic proposal in 1986. After twenty years in the doldrums, Senechal wrote for the American Mathematical Society, "What is a quasicrystal?" The paper began, "The short answer is no one [knows]" [16]. She was mistaken: I had by then discovered the quasi-structure factor and metric [2] [3] [6]. These were based on phase-contrast, optimum-defocus images [17] which identify the locations of the heavier atom in the $\mathrm{Al}_{6} \mathrm{Mn}$ icosahedral alloy, owing to high atomic scattering factor. Knowing all of the microscope magnification, the image pattern, and the diffraction pattern, it was obvious that the unit cell is $\mathrm{Al}_{12} \mathrm{Mn}$, with stoichiometry $\mathrm{Al}_{6} \mathrm{Mn}$ because of edge sharing. These cells are hierarchically arranged, each order having 6 five-fold rotation axes. In particular, four tiers of hierarchic structure are evident in the data and this structure was shown to be infinitely extensible: it is logarithmically periodic with period $\tau^{2}$, the square of the golden section $\tau=\left(1+5^{1 / 2}\right) / 2$ [18].

To calculate the diffraction pattern from the known structure, it was necessary to correctly index [9] the original diffraction pattern [1] [2]. From stereographic projections of the icosahedral axes and diffraction planes, the indexation was shown to be three dimensional, in geometric series, simple and complete. The planes are normal to the three-dimensional axes. Mathematicians choose six dimensions; physicists falsify them.

A breakthrough in understanding of the diffraction pattern followed our realization that the structure factor is independent of scattering angle and can be simply calculated from our knowledge of the structure [2] [14]. Prima facie, the structure factor was inconsistent with data. This inconsistency was half expected because of known "quasi-periodicity", so we included two adjustments to the formulae. Firstly, a coherence term $c_{s}$ which has the effect similar to making the structure breath so that the quasi-Bragg condition became evident by sudden diffractive coherence, that is similar to Bragg coherence observed by rotation of a crystal through the Bragg condition: suddenly on and suddenly off. Bragg diffraction is bi-planar where quasi-Bragg diffraction is multi-planar [14], but the breathing coherence provides sharp diffraction at the quasi-Bragg condition [12], in spite of the "quasiperiodic lattice". Actually of course, there is no mechanical breathing strain; the coherence is due to axial contraction owing to the peculiar hierarchic optics as will be described below by a new law in physics. Secondly, it became obvious that because our unit cells do not repeat periodically in linear order, we had to sum our quasi-structure not just over the unit cell as in crystals; but over the whole quasicrystal (QC) in hierarchic order. The quasi-structure factor formula (QSF) allowed an important iterative procedure that was necessary for the summation of high orders: the iteration overcame computing truncation errors. The final result is consistent with experimental diffraction data [2] [5]. An example will be given in Figure 1. This shows four peaks in 
geometric series, offset from the Bragg condition by the metric 0.894 . This will be elaborated in the next section.

The outstanding question remained, what is the metric and how does the geometric series diffraction occur? It is not consistent with Bragg diffraction; but rather diametrically opposed by geometric, aperiodic, and anharmonic orders $n$, and multiple spacings $d$ at any given Bragg condition. The new quasi-Bragg law was evident: $\tau^{m} \lambda=2 d^{\prime} \sin \left(\theta^{\prime}\right)$, where through multiple simulations, the role of the metric defined all of the lattice parameter $a$; the order $n^{\prime} \rightarrow m$; the quasi-interplanar spacing $d^{\prime}$; and quasi-Bragg angle $\theta^{\prime}$ (the compliment to the angle of incidence). After completely understanding the metric in this unique diffraction, the varieties of data verify both the structure and the diffraction.

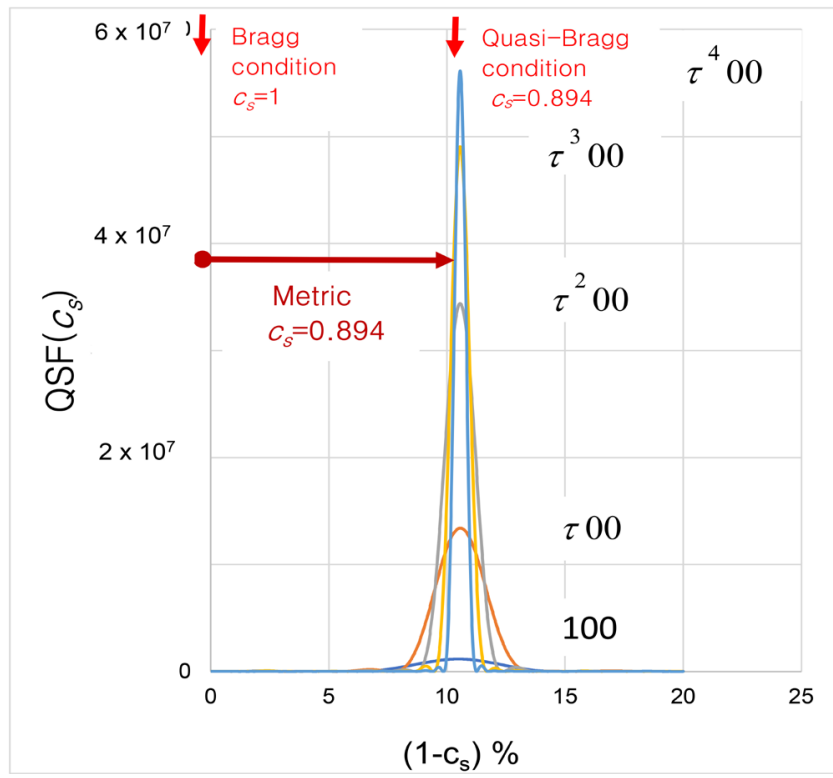

Figure 1. Quasi structure factors calculated by scanning for five peaks in geometric series against the coherence factor $c_{s}$ [14]. At the Bragg condition, $c_{s}=1$ (ordinate axis), and there is no diffraction; the diffraction peaks occur at the quasi-Bragg condition when the metric $C_{\mathrm{s}}=0.894$. All peaks in the diffraction pattern of ref. [1] are calculated to occur at this condition. Geometric series indices are shown level with the tops of corresponding QSF peaks.

\section{Quasi Structure Factor (QSF) Suppresses Diffraction}

The sites of atoms and cell centers in icosahedral clusters are known [2] [13] [14], and also sites of higher order $p$ of supercluster centers, where radii multiply by $\tau^{2 p}:$

$$
\begin{aligned}
\text { Unit cell }\left(r_{u}\right): & \text { Mn } \\
\text { Al } & \frac{1}{2}( \pm \tau, 0, \pm 1), \frac{1}{2}(0, \pm 1, \pm \tau), \frac{1}{2}( \pm 1, \pm \tau, 0)
\end{aligned}
$$

and

$$
\text { Cell or cluster centers }\left(r_{c c}\right) \quad \frac{1}{2}\left( \pm \tau^{2}, 0, \pm \tau\right), \frac{1}{2}\left(0, \pm \tau, \pm \tau^{2}\right), \frac{1}{2}\left( \pm \tau, \pm \tau^{2}, 0\right)
$$


The QSF formula is adapted from classical crystallography with two differences:

$$
F_{h k l}=\sum_{i=1}^{\text {all atoms }} f_{i} \cos \left(2 \pi \cdot c_{s}\left(\overline{h_{h k l}} \cdot \overline{r_{i}}\right)\right)
$$

firstly, because the diffraction is sharp in spite of multiple interplanar spacings $d$, a coherence factor $c_{s}$ is inserted. Its value will be derived analytically below. The factor is used as a scanned variable (Figure 1) to illustrate the variance of quasi-Bragg diffraction from Bragg diffraction in crystals.

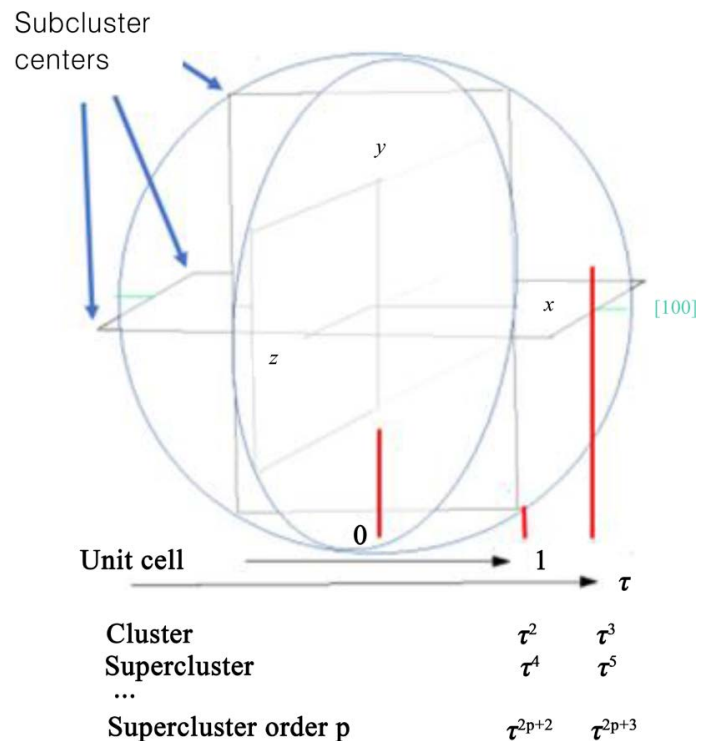

Figure 2. Each golden triad has three principal planes on each dimension that intersect (red pointers) at normals on the [100] axis in the diagram. Each corner of each golden rectangle locates the center of a cell or subcluster. Intercluster spacings are arranged at intervals $0,1, \tau$, for the unit cell. By scaling the triad for clusters and superclusters, the spacings continue $\tau^{2}, \tau^{3}, \tau^{4}, \cdots$ in geometric series, as observed in diffraction indices.

Secondly, because the unit cells are not periodic as in crystals, the summation is made over all atoms in the QC; not just the unit cell. The summation is taken in two steps: over the unit cell and cluster, and iteratively over the superclusters in hierarchic order $p$. Write the vector from the origin to each atom in a cluster $\overline{r_{c l}}$ as the sum of a unit cell vector $\overline{r_{u}}$, with a vector to the cell centers in the cluster $\overline{r_{c c}}: \overline{r_{c l}}=\overline{r_{c c}}+\overline{r_{u}}$. Then since:

$$
\sum_{i}^{N_{\text {cluster }}} \exp \left(\overline{h_{h k l}} \cdot \overline{r_{c l}}\right)=\sum_{i}^{12} \exp \left(\overline{h_{h k l}} \cdot \overline{r_{c c}}\right) \times \sum_{i}^{13} \exp \left(\overline{h_{h k l}} \cdot \overline{r_{u}}\right)
$$

with corresponding summations over unit cell sites and cell centers, and knowing that $N_{\text {cluster }}=N_{c c} \cdot N_{u}$, the QSF for the cluster is calculated:

$$
F_{h k l}^{\text {cluster }}=\sum_{i=1}^{12} \cos \left(2 \pi \cdot c_{s}\left(\overline{h_{h k l}} \cdot \overline{r_{c c}}\right)\right) \cdot F_{h k l}^{\text {cell }}
$$

and repeating iteratively over superclusters by using the known stretching factor $\tau^{2 p}$ : 


$$
F_{h k l}^{p}=\sum_{i=1}^{\text {all atoms }} \cos \left(2 \pi \cdot c_{s}\left(\overline{h_{h k l}} \cdot \tau^{2 p} \overline{r_{c c}}\right)\right) \cdot F_{h k l}^{p-1}
$$

The example in Figure 1 is for a simple axial series, but all beams in the original data [1] display the same metric deviance from the Bragg condition on the ordinate axis where $c_{s}=1$. In the QCs all beams peak at the quasi-Bragg condition $c_{s}=0.894$. There is no Bragg diffraction. From larger samples, the value of $c_{s}$ is extremely precise [5]. As we shall see, this value is the result of harmonization of the incident, sine wave probe with the aperiodic, hierarchic structure. Notice that the QSF depends on the angle between a specific atomic plane-normal and the crystal structure; it is independent of the experimental Bragg angle. Elimination of the Bragg angle $\theta$ from the equation, allowed access to parameters $n$ ', $d$ ', and hierarchic lattice parameter $a$. (The value of the QSF depends greatly on the number of atoms in the simulated quasicrystal as also partly on atomic scattering factors: the number is much larger and more varied than the number of atoms in a crystal unit cell).

\section{Stretching the Axis for Bloch}

Bragg diffraction is bi-planar: the path difference between two rays reflected from neighboring Bragg planes is equal to the wavelength of light. QC diffractions multiplanar, as is observed in high resolution electron micrographs: within the "quasi-periodic solids" every atom scatters. To know how the phases of the various scattered rays add, it is necessary to calculate the QSF. The addition is iterative (Equation (6)). Subclusters locate on the corners of the golden rectangles shown in Figure 2, i.e. on principal planes. These planes, in hierarchic diffraction, replace Bragg planes in diffraction from crystals. Keeping the golden triad as basis in the hierarchy, the spacings between principal planes scale geometrically owing to the stretching factor $\tau^{2 p}$ that determines axial dimensions with increasing order.

Interplanar spacings are ordered like the diffraction pattern: $0,1, \tau, \tau^{2}, \tau^{3}, \tau^{4}, \tau^{5}, \cdots$ It is evident that whereas Bragg diffraction occurs by coherent scattering from Bragg planes, hierarchic diffraction occurs by coherent scattering from subcluster centers. How, more precisely, this happens will be illustrated with quasi-Bloch waves. These waves differ from both the Bloch wave in crystals and from the Bragg diffracted wave beam. Bloch waves are evident as lattice images in the two-beam condition [19]. The waves are due to interference between an incident X-ray or electron beam with its specularly reflected diffraction beam. However, because the interplanar spacings are not in linear order in the QC, an imagined pseudo-Bragg Bloch wave (blue waves in Figure 3) may be coherent in the unit cell but must then be incoherent with the geometric, principal-plane, hierarchic lattice that describes the higher order icosahedra. The pseudo interference is destructive. To construct interference, the interfering sine wave must be stretched by the inverse of the metric. This makes the quasi-Bloch wave commensurate and approximately harmonic with the principal planes. The interfe- 
rence is accompanied by a small (10.6\%) change in scattering angle, away from the Bragg condition i.e. smaller than would occur in crystals having equivalent interplanar spacing. The result is the quasi-Bragg angle $\theta^{\prime}$ to be used below in the quasi-Bragg law. Before we reach for this conclusion, we must explain the origin for the metric that was calculated in the QSFs, and that is critically needed to find the function $f\left(n^{\prime}, d^{\prime}, \theta^{\prime}, c_{s}\right)=0$ for the quasi-Bragg diffraction. The strong explanation is principally numerical, and will be described in the next section.

Meanwhile, it is obvious in Figure 3, that whereas long range order is evident from the diffraction of quasicrystals, it is not true that there is no translational symmetry: the quasi-Bloch wave is invariant in all translations $a \tau^{m}$. There is additional symmetry because the hierarchic model is also centrosymmetric. Notice the extraordinary feature: the quasi-Bloch wave symmetry is both long range and local at each intercept in Figure 3(b).

(a)

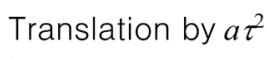

(b)
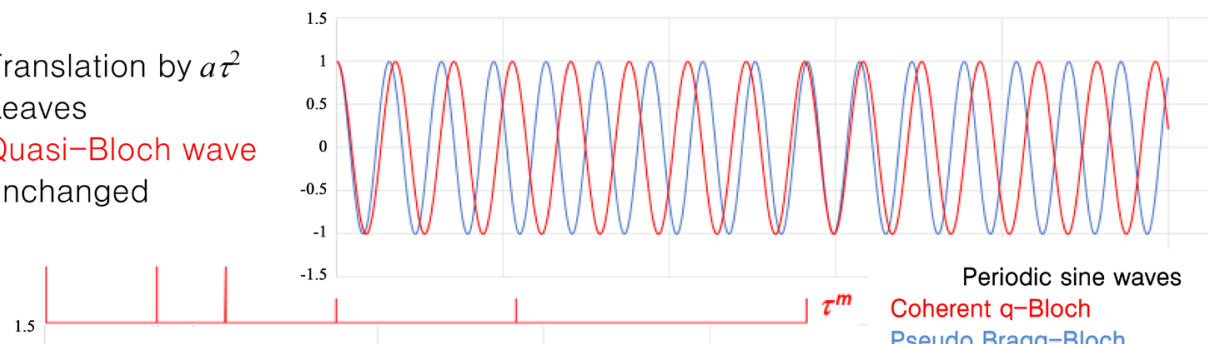

$\tau^{m} \quad$ Coherent q-Bloch

Periodic sine waves Pseudo Bragg-Bloch

(c)

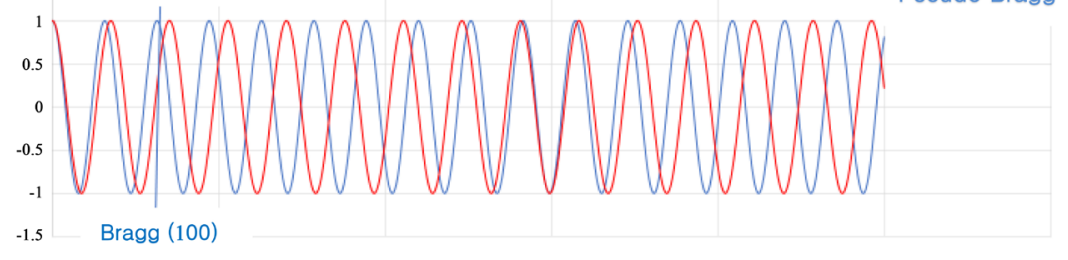

(d)

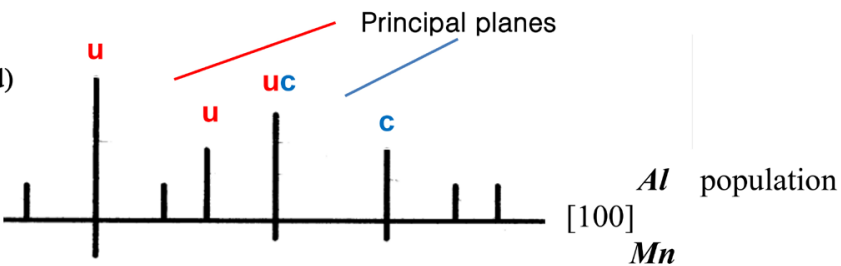

Figure 3. (a) Red Quasi-Bloch wave invariant under translation $a \tau^{m}$ compared with blue pseudo-Bragg Bloch wave that is incommensurate with the structure below in d. (b) Geometric series that mark locations of principal plane intercepts in the unit cell and cluster as below. (c) Same traces as (a), but translated to demonstrate local and long range invariance of the quasi-Bloch wave, and approximate coherence with structure. (d), Atomic planes in the semi-cluster that cross the [100] axis, including principal planes: $\mathrm{u}$ for the unit cell; $\mathrm{c}$ for the cluster. Where the pseudo-Bragg Bloch wave is incoherent.

\section{Irrational Metric Function}

Column 7 in Table 1 lists the geometric series, base $\tau$. Corresponding values are shown in col.9. They are irrational excepting the first. The exact Fibonacci equivalents are shown in cols $2-5$. They can be rationalized by replacing the irrational number $t$ by the fraction 3/2. For harmony, the fraction should be 
Table 1. The bold type in column 7 shows the geometric series that correctly indexes the observed diffraction pattern. Corresponding irrational values are shown in col. 9 and the common Fibonacci sequence (cols 2 - 5), which sums a rational part and an irrational part. By substituting the rational fraction 3/2 for irrational $\tau$ in col.5, the rational series in col.10 is derived. QSFs calculated for this imaginary structure are Bragg-like with $C_{s}=1$. This fact confirms the supposition that the metric, calculated in the quasi-Bragg QSFs, is due to the irrational part of col.9 (i.e. completely absent in Bragg diffraction). Subtract it from col.9 and harmonize the residue by dividing col. 11/col. 12 (corresponding to harmonics in Figure 3). Derive the metric in the final col. 14 . This value is identical to the metric simulated universally in QSFs. N.B. $\tau^{m}=F_{m}(1, \tau)=F_{m}(0,1)+F_{m+1}(0,1) \tau$; where $m>0$; ratios $F_{m+1}(0,1) / F_{m}(0,1)$ oscillate about $\tau$, contrasting $F_{m+1}(1, \tau) / F_{m}(1, \tau)=\tau$, as in the diffraction data.

\begin{tabular}{|c|c|c|c|c|c|c|c|c|c|c|c|c|c|}
\hline & & \multicolumn{4}{|c|}{ Fibonacci series } & \multicolumn{2}{|c|}{$\begin{array}{l}\text { Geometric } \\
\text { series }\end{array}$} & $\begin{array}{l}\text { Irrational } \\
\text { values }\end{array}$ & $\begin{array}{l}\text { Rational } \\
\text { Approx. }\end{array}$ & $\begin{array}{l}\text { Irr.-Rat. } \\
\text { residue }\end{array}$ & $\begin{array}{c}\text { Commensurate } \\
\text { divisor }\end{array}$ & $\begin{array}{l}\text { Residue/ } \\
\text { divisor }\end{array}$ & Metric \\
\hline \multirow{3}{*}{$m$} & & & & & & $\tau^{m-1}$ & & & & & & & $C_{\mathrm{s}}$ \\
\hline & $F_{m}$ & & $F_{m+1}$ & $\tau$ & & & & & $a+3 b / 2$ & & $/ F_{m+1}$ & $\Delta=\tau-3 / 2$ & $1 /(1+\Delta)$ \\
\hline & & & 0 & $\tau$ & & $\tau^{0}$ & $=$ & 1 & 1 & 0 & 0 & & \\
\hline 1 & 0 & & 1 & $\tau$ & $=$ & $\tau$ & $=$ & $1.618034 \ldots$ & 1.5 & 0.11803 & 1 & 0.118034 & 0.894427 \\
\hline 2 & 1 & + & 1 & $\tau$ & $=$ & $\tau^{2}$ & $=$ & $2.618034 \ldots$ & 2.5 & 0.11803 & 1 & 0.118034 & 0.894427 \\
\hline 3 & 1 & + & 2 & $\tau$ & $=$ & $\tau^{3}$ & $=$ & $4.236068 \ldots$ & 4 & 0.23607 & 2 & 0.118034 & 0.894427 \\
\hline 4 & 2 & + & 3 & $\tau$ & $=$ & $\tau^{4}$ & $=$ & 6.854102 & 6.5 & 0.3541 & 3 & 0.118034 & 0.894427 \\
\hline 5 & 3 & + & 5 & $\tau$ & $=$ & $\tau^{5}$ & $=$ & 11.09017 & 10.5 & 0.59017 & 5 & 0.118034 & 0.894427 \\
\hline 6 & 5 & + & 8 & $\tau$ & $=$ & $\tau^{6}$ & $=$ & 17.944272 & 17 & 0.94427 & 8 & 0.118034 & 0.894427 \\
\hline 7 & 8 & + & 13 & $\tau$ & $=$ & $\tau^{7}$ & $=$ & 29.034443 & 27.5 & 1.53444 & 13 & 0.118034 & 0.894427 \\
\hline 8 & 13 & + & 21 & $\tau$ & $=$ & $\tau^{8}$ & $=$ & 46.978715 & 44.5 & 2.47872 & 21 & 0.1180341 & 0.894427 \\
\hline 9 & 21 & + & 34 & $\tau$ & $=$ & $\tau^{9}$ & $=$ & 76.013159 & 72 & 4.01316 & 34 & 0.1180341 & 0.894427 \\
\hline 10 & 34 & + & 55 & $\tau$ & $=$ & $\tau^{10}$ & $=$ & 122.99188 & 116.5 & 6.49188 & 55 & 0.1180341 & 0.894427 \\
\hline 11 & 55 & + & 89 & $\tau$ & $=$ & $\tau^{11}$ & $=$ & 199.00504 & 188.5 & 10.505 & 89 & 0.1180341 & 0.894427 \\
\hline 12 & 89 & + & 144 & $\tau$ & $=$ & $\tau^{12}$ & $=$ & 321.99691 & 305 & 16.9969 & 144 & 0.1180341 & 0.894427 \\
\hline
\end{tabular}

integral or half integral. QSF calculations show it is half integral. The rational approximation to the geometric series indices is listed in col. 10. Calculation of the QSFs for this imaginary series is Bragg-like with $c_{s}=1$. This demonstrates the fact that the metric is an expression of the irrational part of the geometric sequence in col. 7. To derive the metric, subtract the rational part from the irrational sequence (col. 7 - col. 10) to give the residue in column 11. Notice that this is a growing number down the sequence, and that it can be harmonized by the integers $F_{m+1}(0,1)$ in col. 12 , i.e. the Fibonacci sequence $F_{m+1}=0,1,1,2,3,5, \cdots$, with the argument representing the first two terms. The re-normalization corresponds to the increasing number of periods between intercepts illustrated in Figure 3. Division of the irrational residue by this sequential harmonization leaves the constant, irrational number. When this is represented as an increment on the $c_{s}=1$ at the Bragg condition, and then inverted, we derive, from the irrational 
indices, the analytic metric which is exactly the same as was discovered, by the perfectly independent numerical method of QSF simulations on the QC. The exact and identical values make the probability for error infinitesimally small and practically impossible.

The result is summarized in Figure 4. The "metric function" is the inverse of the metric and is equal to:

$$
\begin{aligned}
1 / c_{s} & =1+(\tau-3 / 2)=1+0.118034=1 / c_{s} \\
& =1+\left(\tau^{2}-5 / 2\right) \\
& =\frac{1+\left(\tau^{3}-4\right)}{2} \\
& =\frac{1+\frac{\left(\tau^{4}-13 / 2\right)}{3}}{3} \\
& =1+\frac{\left(\tau^{5}-21 / 2\right)}{5} \\
& =1+\frac{\left(\tau^{6}-34 / 2\right)}{8} \\
& \cdot \quad \frac{\text { Irrational rational semi-integer }}{\text { subtraction }} \\
= & 1+\left(\frac{\left(\tau^{m}-F_{m+4} / 2\right)}{F_{m+1}}=\frac{1}{0.894} \quad \begin{array}{c}
\text { : Metric } \\
\text { function }
\end{array}\right.
\end{aligned}
$$

Figure 4. The metric function is derived by subtraction of the rational part from the irrational index $F_{m}(1, \tau)=1, \tau, \tau^{2}, \tau^{3}, \cdots$ (see Table 1 ). After division by a commensurating harmonic number, $F_{m+1}(0,1)=0,1,1,2,3,5,8,11, \cdots$, (also Fibonacci, see Figure 3), the metric expresses computational "breathing" of the QC axes that are necessary for coherence. This condition was obtained by scanning $C_{s}$ to find the maximum QSF. The metric function is the inverse of the metric that is simulated universally in QSFs. The first six terms are evaluated as examples. The wave expands when the metric contracts the axes.

$$
\frac{1}{C_{s}}=1+\frac{\tau^{m}-F_{m+4} / 2}{F_{m+1}}=\frac{1}{0.894}
$$

To illustrate, the first six terms are shown in the figure: the metric is exactly derived from the irrational part of the index, the part that is completely absent in the Bragg formula.

The metric may be derived in several ways, one is as follows: An index $\tau^{m}$ is separated into rational and irrational parts while $\tau$ is separated into the rational semi-integral 3/2 and an irrational residue $\Delta=\tau-3 / 2$ (Table 1):

$$
\begin{aligned}
\tau^{m} & =F_{m}+F_{m+1} \cdot \tau \\
& =F_{m}+F_{m+1}+F_{m+1} / 2+F_{m+1} \cdot \Delta \\
& =F_{m+2}+F_{m+1} / 2+F_{m+1} \cdot \Delta \\
& =F_{m+4} / 2+F_{m+1} \cdot \Delta
\end{aligned}
$$

Since, by general properties of the Fibonacci sequence:

$$
\begin{aligned}
F_{m+4} / 2 & =F_{m+3} / 2+F_{m+2} / 2 \\
& =F_{m+2} / 2+F_{m+1} / 2+F_{m+2} / 2 \\
& =F_{m+2}+F_{m+1} / 2
\end{aligned}
$$


Equation (8) is thereby systematically confirmed from Table 1 and Figure 4, and rearranged:

$$
\Delta=\frac{\tau^{m}-F_{m+4} / 2}{F_{m+1}}
$$

So that the metric function:

$$
\frac{1}{C_{S}}=1+\Delta=\frac{1}{0.894}
$$

in complete agreement with the QSF simulations where $c_{s}=1 / 1.1180$, and $\Delta=\tau-3 / 2=0.1180$. This irrational part (Table 1, col. 11), can be written $F_{m+1}(1, \tau-3 / 2) \Delta$, consistent with Equation (8).

The metric can be derived in further ways: notice, for example, that if $\tau$ is supposed to vary, then $c_{s} \rightarrow 1$ as $\tau \rightarrow 0$, i.e. at the Bragg condition. Generally however, the metric commensurates and harmonizes the diffracted sine wave onto a geometric grid [2] [14], as is simulated in the QSFs.

A summary of the structural result is shown in Figure 5. Notice, in particular, that the lattice parameter for the QC that was measured a long time ago on an assumption of Bragg diffraction [20] [21], is now corrected for the metric and index under the quasi-Bragg law: $a=0.205 \cdot \tau \cdot c_{s} \mathrm{~nm}$. Within errors, $a$ is equal to both the known diameter of $A l$ and to the edge width of the unit cell (Figure 6). To understand this, consider the edge of the unit cell. Twelve $A I$ atoms are closely packed around the central $M n$ atom. The edge width of the unit cell is the sum of the radii of two $A l$ atoms [5] [14] [15]. The experimental value of $a$ is a necessary consistency test for any model that has been proposed, and the test is only possible with a systematic and complete theory of the unique diffraction. There is a further fact beyond the strong evidence from imaging and

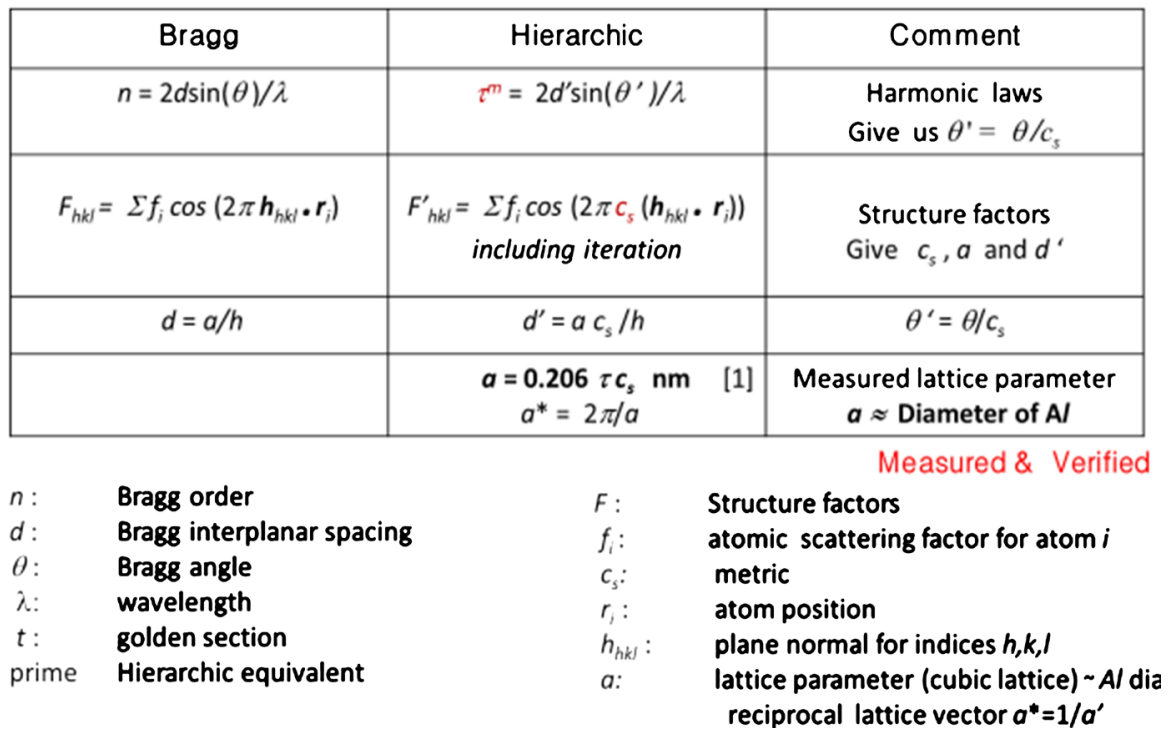

Figure 5. Comparison of Bragg parameters in crystals with quasi-Bragg parameters in quasicrystals. Notice especially the corrections (col. 2, row 5) to the lattice parameter $a$, derived from the earlier, false assumption of Bragg diffraction. 


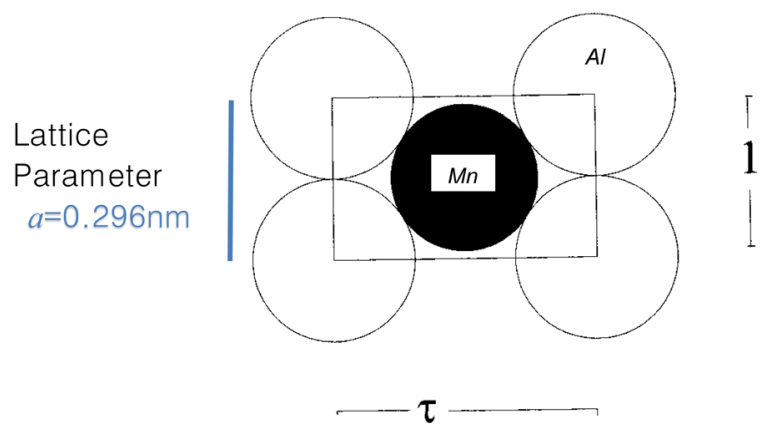

Figure 6. Cross-section of icosahedral unit cell showing central $\mathrm{Mn}$ atom touching outer $A l$ atoms centered on corners of the golden rectangle. The measured lattice parameter, $a=0.296 \mathrm{~nm}$, equals the width of the golden rectangle and the diameter of $A l$. The dense unit cell has 15 identical cross-sections between the 30 edges on the Platonic solid. Alongside the imaging of hierarchies and theory of diffraction, the measurement verifies the structure.

diffraction, namely that all diatomic, icosahedral QCs have atomic diameter ratios: $D_{\text {solute }} / D_{\text {solvent }}=\left(1+\tau^{2}\right)^{1 / 2}-1$, as indeed do $D_{\mathrm{Mn}} / D_{\mathrm{Al}}$ in the figure. The fact is consistent with high local density as the structural driving force.

The true measurement of the lattice parameter, with the correction given by the metric and index under the quasi-Bragg law (Figure 5), verifies the structure and diffraction of the QC.

\section{Welcome Hierarchic Physics}

The quasicrystal has inter-related icosahedral symmetry with diffraction in geometric series: an incident X-ray or electron beam scatters off the hierarchic lattice into geometric space. The metric, that is measured and experimentally verified, is now completely understood. This is unique and novel in QCs. Quasicrystallographers have, for 38 years refused to accept this fact, though some, like Senechal have acknowledged shortcomings. For example, a sub-editor of Acta Crystallographica wrote that you don't measure the lattice parameter, "You just have to choose ' $d_{h}$ ' ", the interplanar spacing [[5] p. 82]. This is a category error: mathematicians choose their axioms; physicists falsify them [22] [23]. He went on to write, "Bragg's equation cannot be applied if we do not know how to handle the term $d_{h}$ ". He doesn't "know" since he "chooses" $a$ in $d_{h k l}=a / m \cdot\left(h, k, l^{2}\right)$ falsely and inaccurately (without metric); while Bragg's law never applies. His is first rank, mainstream, quasi-physics. As we have shown, the dimensions that mathematicians invent are multiplied without necessity; it is ironic that the metric function supplies the new dimensionality that they sought. Whatever, they do not review papers objectively when their own writings and readings are made irrelevant. The formal and informal logic that has guided science for two millenia are observed by neglect, and this is evident in published reviews. Other mathematical theories are equally quasi [23] [24], as Feynman famously observed, "No one understands quantum mechanics". But obviously, whatever is not understood is not physics. 


\section{Conclusion}

The quasi-Bragg law is a new law in physics. Now that the structure and diffraction are clear for anybody to see and understand, we should turn our attention to outlying problems. These include quasicrystalloids in which planar five-fold, six-fold and eight-fold quasicrystal symmetries link to regular linear symmetries on planar normals. The icosahedral unit cell may share edges in various ways, and metrics are likely dependent on them individually. Another class of problems is defects, especially in rapidly quenched material. Some anomalous microstructures have been recorded, by convergent beam diffraction for example [21], so these, along with grain boundaries [14], might give important information about hierarchic crystal growth. Supposing that the dense unit cell nucleates in the melt before solidification, it is not obvious how clusters, superclusters and higher orders grow and agglomerate during solidification. It may become necessary to discover methods that can be used on samples that are more bulky than thin foils observed in typical transmission electron microscopy. Hierarchic crystals present ever more interesting phenomena, where mature and complete understanding now graduates them as a new field in physics.

\section{Conflicts of Interest}

The author declares no conflicts of interest regarding the publication of this paper.

\section{References}

[1] Shechtman, D., Blech, I., Gratias, D. and Cahn, J.W. (1984) Physical Review Letters, 53, 1951-1953. https://doi.org/10.1103/PhysRevLett.53.1951

[2] Bourdillon, A.J. (2009) Quasicrystals and Quasi Drivers. UHRL, San Jose.

[3] Bourdillon, A.J. (2011) Logarithmically Periodic Solids. Nova Science, New York.

[4] Bourdillon, A.J. (2010) Quasicrystals' 2D Tiles in 3D Superclusters. UHRL, San Jose.

[5] Bourdillon, A.J. (2012) Metric, Myth and Quasicrystals. UHRL, San Jose, 82.

[6] Bourdillon, A.J. (2009) Solid State Communications, 149, 1221-1225. https://doi.org/10.1016/j.ssc.2009.04.032

[7] Bourdillon, A.J. (2014) Journal of Modern Physics, 5, 488-496. https://doi.org/10.4236/jmp.2014.56060

[8] Bourdillon, A.J. (2014) Journal of Modern Physics, 5, 1079-1084. https://doi.org/10.4236/jmp.2014.512109

[9] Bourdillon, A.J. (2013) Micron, 51, 21-25. https://doi.org/10.1016/j.micron.2013.06.004

[10] Bourdillon, A.J. (2016) Journal of Modern Physics, 7, 43-50. https://doi.org/10.4236/jmp.2016.71005

[11] Bourdillon, A.J. (2015) Physical Chemistry \& Biophysics, 5, 3.

[12] Bourdillon, A.J. (2016) Journal of Modern Physics, 7, 1558-1567. https://doi.org/10.4236/jmp.2016.712142

[13] Bourdillon, A.J. (2019) Journal of Modern Physics, 10, 624-634. 
https://doi.org/10.4236/jmp.2019.106044

[14] Bourdillon, A.J. (2019) Journal of Modern Physics, 10, 1364-1373.

[15] Bourdillon, A.J. (2020) Complete Solution for Quasicrystals. https://www.youtube.com/watch?v=OFcSDKCecDA

[16] Senechal, M. (2006) Notices to the American Mathematical Society, 3, 886-887.

[17] Bursill, L.L. and Peng, J.L. (1985) Nature, 316, 50-51. https://doi.org/10.1038/316050a0

[18] Huntley, H.E. (1970) The Divine Proportion. Dover, Mineola.

[19] Hirsch, P., Howie, A., Nicholson, R.B., Pashley, D.W. and Whelan, M.J. (1977) Electron Microscopy of Thin Crystals. Krieger, New York.

[20] Tsai, A.P. (2008) Science and Technology of Advanced Materials, 9, 1-20. https://doi.org/10.1088/1468-6996/9/1/013008

[21] Bourdillon, A.J. (1987) Philosophical Magazine Letters, 55, 21-26. https://doi.org/10.1080/09500838708210435

[22] Popper, K.R. (1959) The Logic of Scientific Discovery. Hutchinson, London. https://doi.org/10.1063/1.3060577

[23] Popper, K.R. (1982) Quantum Theory and the Schism in Physics. Hutchison, London.

[24] Bourdillon, A.J. (2019) Journal of Modern Physics, 11, 365-377. https://doi.org/10.4236/jmp.2019.106044 